\title{
THE SMOLYANOV SURFACE MEASURE ON TRAJECTORIES IN A RIEMANNIAN MANIFOLD
}

\author{
NADEZDA A. SIDOROVA \\ Moscow State University, Faculty of Mechanics and Mathematics, \\ 119899 Moscow, Russia \\ Mathematical Institute, University of Oxford, \\ 24-29 St Giles' Oxford OX1 3LB, UK \\ sidorova@maths.ox.ac.uk \\ Received 9 February 2004 \\ Communicated by L. Accardi
}

\begin{abstract}
It has been shown in Refs. 2-6 that two natural definitions of surface measures, on the space of continuous paths in a compact Riemannian manifold embedded into $\mathbb{R}^{n}$, introduced in the paper by Smolyanov ${ }^{1}$ are equivalent; this means that there exists a natural object - the surface measure, which we call the Smolyanov surface measure. Moreover, it has been shown ${ }^{2-6}$ that this surface measure is equivalent to the Wiener measure and the corresponding density has been found. But the known proof of the equivalence of the two definitions of the surface measure is rather nonexplicit; in fact the densities of the measures corresponding to the two different definitions were found independently and only a posteriori it was discovered that those densities coincided.

Our aim is to give a direct proof of this fact. We introduce a more restrictive definition of the surface measure as the weak limit of a standard Brownian motion in $\mathbb{R}^{n}$ conditioned to be in the tubular $\varepsilon$-neighborhood of the manifold at times $0=t_{0}<t_{1}<\cdots<t_{n-1}<t_{n}=1$ as both $\varepsilon$ and the diameter of the partition tend to zero. Letting $\varepsilon$ and then the diameter of the partition tend to zero and vice versa, we arrive at the two definitions above. We prove the existence of the Smolyanov surface measure using our definition, show that this measure is equivalent to the law of a Brownian motion on the manifold, and compute the corresponding density in terms of the curvature of the manifold. As a special case of this, we again obtain the results of Refs. $2-6$.
\end{abstract}

Keywords: Surface measures; Brownian motion; Riemannian manifolds.

AMS Subject Classification: 58J65, 60J65, 60B10

\section{Introduction}

The surface measures on submanifolds having both infinite dimension and codimension were introduced by Smolyanov in $1995 .{ }^{1}$ He considered the space of continuous paths in $\mathbb{R}^{n}$ equipped with the Wiener measure as an ambient space and 
the subspace of continuous paths in a compact Lie grop $G \subset \mathbb{R}^{n}$ as a submanifold. ${ }^{\text {a }}$ In fact, that paper introduced the two different definitions of the surface measure mentioned above and it was noted that (in this case), both measures coincide with the Wiener measures on trajectories in the Lie group $G$. Both definitions can also be applied to the case when the Lie group is replaced with any Riemannian manifold. For that more general case the surface measures were investigated in Refs. 2-6.

Let $M$ be a compact Riemannian manifold and $a_{0} \in M$. Denote by $C_{a_{0}}\left([0,1], \mathbb{R}^{n}\right)$ the space of continuous real-valued functions on $[0,1]$ taking value $a_{0}$ at zero, and by $C_{a_{0}}([0,1], M)$ its subset consisting of $M$-valued functions.

One of the natural ways ${ }^{1}$ to define a surface measure on $C_{a_{0}}([0,1], M)$ corresponding to the Wiener measure $\mathbb{W}$ on the ambient space is to force the Brownian particle to be in the manifold at times $0=t_{0}<t_{1}<\cdots<t_{n-1}<t_{n}=1$ and to let the mesh of the partition $P$ go to zero. More precisely, the surface measure is defined as the weak limit

$$
\mathbb{S}_{1}=\lim _{|P| \rightarrow 0} \mathbb{W}\left(\cdot \mid \omega_{t_{i}} \in M \text { for all } t_{i} \in P\right) .
$$

With the help of explicit estimates for the short time asymptotics of the conditional integral operator and using the semigroup theory it has been proved ${ }^{2,3,5}$ that $\mathbb{S}_{1}$ exists, is equivalent to the Wiener measure $\mathbb{W}_{M}$ on $C_{a_{0}}([0,1], M)$, and the density has been computed explicitly in terms of the mean and scalar curvature of the manifold.

The second way ${ }^{1}$ to define a surface measure on $C_{a_{0}}([0,1], M)$ corresponding to the standard Brownian motion on $\mathbb{R}^{n}$ is to condition on the event that the Brownian particle does not leave the tubular $\varepsilon$-neighborhood $\mathbb{M}_{\varepsilon}$ of $M$ for the whole time, and then let $\varepsilon$ go to zero, i.e.

$$
\mathbb{S}_{2}=\lim _{\varepsilon \rightarrow 0} \mathbb{W}\left(\cdot \mid \omega_{t} \in \mathbb{M}_{\varepsilon} \text { for all } t \in[0,1]\right) .
$$

It has been proved ${ }^{4,6}$ using mainly SDE techniques that $\mathbb{S}_{2}$ also exists, is equivalent to $\mathbb{W}_{M}$ and the density appears to be the same as of $\mathbb{S}_{1}$ (although there was no direct proof that $\mathbb{S}_{1}=\mathbb{S}_{2}$ ).

In the present paper we introduce a more restrictive definition of the surface measure. Namely, let

$$
\mathbb{W}_{P, \varepsilon}=\mathbb{W}\left(\cdot \mid \omega_{t_{i}} \in \mathbb{M}_{\varepsilon} \text { for all } t_{i} \in P\right)
$$

and

$$
\mathbb{S}=\lim _{\substack{|P| \rightarrow 0 \\ \varepsilon \rightarrow 0}} \mathbb{W}_{P, \varepsilon}
$$

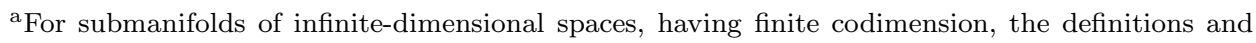
properties of surface measures can be (relatively) easily obtained in the frame of the theory of differentiable measures initiated by Fomin (see Ref. 12) (where in particular the notion of logarithmic derivative of a measure has been introduced). In this framework the theory of surface measures on submanifolds of finite codimension has been developed by Uglanov ${ }^{16}$ (see also Refs. 13 and 15).
} 
It is easy to see that

$$
\mathbb{S}_{1}=\lim _{|P| \rightarrow 0} \lim _{\varepsilon \rightarrow 0} \mathbb{W}_{P, \varepsilon} \quad \text { and } \quad \mathbb{S}_{2}=\lim _{\varepsilon \rightarrow 0} \lim _{P \mid \rightarrow 0} \mathbb{W}_{P, \varepsilon}
$$

We prove the existence of $\mathbb{S}$ (which implies the existence of $\mathbb{S}_{1}$ and $\mathbb{S}_{2}$ ) and compute its density with respect to $\mathbb{W}_{M}$ (which must certainly be the same as of $\mathbb{S}_{1}$ and $\mathbb{S}_{2}$ ) in an easier way.

Let us also notice that in Refs. 9, 10, it is shown that the definition of $\mathbb{S}_{1}$ is well adapted to obtaining the Feynman-Kac type formulas for Schrödinger equations on manifolds and the definition of $\mathbb{S}_{2}$ is adapted to develop some change of variable formulas for Wiener measures and Feynman pseudomeasures on trajectories in manifolds. In particular the same density appears in the context of the study of the holonomic constraints in quantum mechanics. ${ }^{8-10,14}$ Hence the geometric potential in the density can be interpreted as an additional effective potential in the Schrödinger equation on the manifold. ${ }^{8-11}$

We use the following notation. We assume that $\varepsilon_{0}$ is small enough and the orthogonal projection $\pi: \mathbb{M}_{\varepsilon_{0}} \rightarrow M$ is well defined (and we consider $\varepsilon<\varepsilon_{0}$ ). We denote by $T_{a} M$ the tangent space of $M$ at $a \in M$ and by $N_{a} M$ the normal space of $M$ at $\pi(a)$, for $a \in \mathbb{M}_{\varepsilon_{0}}$. In the sequel, we identify these spaces with the corresponding subspaces in $\mathbb{R}^{n}$. Further, for each $x \in M$, let $P_{x}$ denote the orthogonal projection of $\mathbb{R}^{n}$ onto $T_{x} M$ and $Q_{x}=I-P_{x}$ denote the orthogonal projection of $\mathbb{R}^{n}$ onto $N_{x} M$. For $x \in M$ and $w \in T_{x} M$, we define

$$
\Gamma_{x}(w)=d Q_{x}(w) P_{x}+d P_{x}(w) Q_{x} \in g l(n) .
$$

We denote by $\mathrm{pr}_{1}: \mathbb{R}^{n} \rightarrow \mathbb{R}^{m}$ (respectively, $\mathrm{pr}_{2}: \mathbb{R}^{n} \rightarrow \mathbb{R}^{k}$ ) the linear operator that maps $u \in \mathbb{R}^{n}$ to its first $m$ (respectively, to its last $k$ ) coordinates and by $\mathrm{pr}_{1}^{-1}$ : $\mathbb{R}^{m} \rightarrow \mathbb{R}^{n}$ (respectively, $\mathrm{pr}_{2}^{-1}: \mathbb{R}^{k} \rightarrow \mathbb{R}^{n}$ ) the right inverse to $\mathrm{pr}_{1}$ (respectively, to $\mathrm{pr}_{2}$ ) such that $\mathrm{pr}_{1}^{-1} \mathrm{pr}_{1}=P_{a_{0}}$ (respectively, $\mathrm{pr}_{2}^{-1} \mathrm{pr}_{2}=Q_{a_{0}}$ ). Besides, we fix an orthonormal basis $\left(e_{i}\right)$ in $\mathbb{R}^{n}$ (such that its first $m$ vectors generate $T_{a_{0}} M$ ) and identify all linear operators on $\mathbb{R}^{n}$ with their matrices with respect to $\left(e_{i}\right)$. We also use the Einstein summation convention: an index occurring twice in a product is to be summed from one up to the space dimension.

Furthermore, we use the notion of the Fermi decomposition of a continuous $\mathbb{M}_{\varepsilon_{0}}$-valued semimartingale. ${ }^{6}$ First, for every such semimartingale $\left(\xi_{t}\right)$ we define $x_{t}=\pi\left(\xi_{t}\right)$. Secondly, we denote by $\left(u_{t}\right)$ the matrix-valued process that performs stochastic parallel translation along $\left(x_{t}\right)$, i.e. $\left(u_{t}\right)$ is a solution of

$$
\delta u_{t}+\Gamma_{x_{t}}\left(\delta x_{t}\right) u_{t}=0 \text { with } u_{0}=I .
$$

Finally, we define $z_{t}=\operatorname{pr}_{2} u_{t}^{T}\left(\xi_{t}-x_{t}\right)$. The triple $\left(x_{t}, u_{t}, z_{t}\right)$ is called the Fermi decomposition of $\left(\xi_{t}\right)$. It is easy to see that $\left(\xi_{t}\right)$ can be reconstructed from its Fermi decomposition by the formula

$$
\xi_{t}=x_{t}+u_{t} \mathrm{pr}_{2}^{-1} z_{t} .
$$


The structure of the proof of our main result is similar to the proof about $\mathbb{S}_{2}$ although each step has undergone a significant change. In Sec. 2 we define the optimal vector field $v$ on $\mathbb{R}^{n}$ (which is in contrast to the shifting vector field ${ }^{6}$ no longer orthogonal to the manifold) and compute its norm and divergence at points on $M$ in terms of the mean and the scalar curvature of $M$. In Sec. 3 we define a new process $\left(y_{t}\right)$ by

$$
\left\{\begin{array}{l}
d y_{t}=d b_{t}-\frac{1}{2} v\left(y_{t}\right) d t \\
y_{0}=a_{0}
\end{array}\right.
$$

which we call a Brownian motion with the optimal drift. Further, writing down the Fermi decomposition $\left(x_{t}, u_{t}, z_{t}\right)$ of $\left(y_{t}\right)$, using the fact that $\left(z_{t}\right)$ turns out to be a $k$-dimensional Brownian motion independent of the Brownian motion driving the SDE for $\left(x_{t}\right)$, and taking into account the continuity of the paths of $\left(y_{t}\right)$, we show that the generalized surface measure corresponding to $\left(y_{t}\right)$ exists and coincides with $\mathbb{W}_{M}$. Finally, in Sec. 4 we prove our main result using the equivalence of the laws of $\left(y_{t}\right)$ and a standard Brownian motion and the continuity of the corresponding density, which is provided by the fact that $v$ is of the gradient type.

\section{Logarithmic Density $\varphi$ and Optimal Vector Field $v$}

In order to define the optimal vector field $v$ mentioned in the Introduction, notice that there are two natural measures $\lambda_{n}$ and $\lambda_{\oplus}$ on $\mathbb{M}_{\varepsilon_{0}}$. The first one is just the Lebesgue measure on $\mathbb{R}^{n}$ restricted to $\mathbb{M}_{\varepsilon_{0}} \subset \mathbb{R}^{n}$. The second one is defined by

$$
\lambda_{\oplus}(A)=\int_{\pi(A)} \lambda_{k}\left(A_{x}\right) d \lambda_{M}(x), \quad A \subset \mathbb{M}_{\varepsilon_{0}} \text { Borel },
$$

where $A_{x}=\pi^{-1}(x)$ and $\lambda_{k}$ and $\lambda_{M}$ are the Lebesgue measures on $\mathbb{R}^{k}$ and $M$, respectively. We have used here the fact that $A_{x} \subset N_{x} M$ and that there is a linear isometry between $N_{x} M$ and $\mathbb{R}^{k}$. Moreover, the Lebesgue measure $\lambda_{k}$ is independent of the choice of such an isometry and hence $\lambda_{\oplus}$ is well-defined.

It has been proved ${ }^{6}$ that $\lambda_{\oplus}$ is equivalent to $\lambda_{n}$. Denote

$$
\varphi=\log \frac{d \lambda_{n}}{d \lambda_{\oplus}}
$$

and define

$$
v=\nabla \varphi
$$

Furthermore, denote by the same symbol a smooth extension of $v$ to $\mathbb{R}^{n}$ that is equal to zero outside a neighborhood of $M$ (the choice of such an extension is not essential).

Definition 2.1. The vector field $v$ is called the optimal vector field. 
Lemma 2.1. For any $a \in M$ we have

$$
\begin{aligned}
& \|v(a)\|^{2}=\|\nabla \varphi(a)\|^{2}=m^{2}\|\kappa\|^{2}(a), \\
& \operatorname{div} v(a)=\Delta \varphi(a)=R(a),
\end{aligned}
$$

where $\kappa$ is the mean curvature vector and $R$ the scalar curvature of $M$.

Proof. Consider an orthogonal coordinate system $\left(x^{1}, \ldots, x^{m}, z^{1}, \ldots, z^{k}\right)$ centered at $a$ such that its first $m$ basis vectors form an orthogonal basis of the tangent space $T_{a} M$. Let us compute $\nabla \varphi(a)$ and $\Delta \varphi(a)$ with respect to this system. By the implicit function theorem, in this coordinate system the manifold $M$ can be represented locally in a neighborhood of the point $a$ by a system of equations $z^{s}=f_{s}\left(x^{1}, \ldots, x^{m}\right)$. Notice that $\partial_{i} f_{s}(0)=0$, for all $i, s$. Further, denote by $F_{s}=$ $\operatorname{Hess} f_{s}(0)$.

It follows from Lemma 9 in Ref. 6 that $\left.\varphi\right|_{M}=0$ and so $\varphi(x, f(x))=0$. Differentiating it with respect to $x^{i}$ and taking into account the equalities $\partial_{x^{i}} f_{s}(0)=0$ we obtain for every $1 \leq i \leq m$

$$
\partial_{x^{i}} \varphi(0,0)=0 .
$$

Again by Lemma 9 in Ref. 6 we obtain for every $1 \leq p \leq k$

$$
\begin{aligned}
\partial_{z^{p}} \varphi(0, z) & =-\partial_{z^{p}} \log \operatorname{det}\left[I-z^{s} F_{s}\right]^{-1} \\
& =\operatorname{tr} \partial_{z^{p}} \log \left[I-z^{s} F_{s}\right]=-\operatorname{tr}\left(\left[I-z^{s} F_{s}\right]^{-1} F_{p}\right) .
\end{aligned}
$$

It follows now from Lemma 5 in Ref. 6 that

$$
\begin{aligned}
\|v(a)\|^{2}=\|\nabla \varphi(a)\|^{2} & =\sum_{i=1}^{m}\left[\partial_{x^{i}} \phi(0,0)\right]^{2}+\sum_{p=1}^{k}\left[\partial_{z^{p}} \phi(0,0)\right]^{2} \\
& =\sum_{p=1}^{k}\left(\operatorname{tr} F_{p}\right)^{2}=m^{2}\|\kappa\|^{2}(a) .
\end{aligned}
$$

Further, differentiating $\varphi(x, f(x))=0$ twice with respect to $x^{i}$ and taking into account $\partial_{x^{i}} f_{s}(0,0)=0$ and $(2.7)$ we obtain

$$
\sum_{i=1}^{m} \partial_{x^{i} x^{i}} \phi(0,0)=-\sum_{p=1}^{k} \partial_{z^{p}} \phi(0,0) \sum_{i=1}^{m} \partial_{x^{i} x^{i}} f_{p}(0)=\sum_{p=1}^{k}\left(\operatorname{tr} F_{p}\right)^{2} .
$$

Finally, it follows from (2.7) that

$$
\sum_{p=1}^{k} \partial_{z^{p} z^{p}} \varphi(0,0)=-\sum_{p=1}^{k} \operatorname{tr}\left(F_{p}\right)^{2}
$$

and Lemma 4 in Ref. 6 implies

$$
\operatorname{div} v(a)=\Delta \varphi(a)=\sum_{p=1}^{k}\left[\left(\operatorname{tr} F_{p}\right)^{2}-\operatorname{tr}\left(F_{p}\right)^{2}\right]=R(a),
$$

which completes the proof. 


\section{Brownian Motion with the Optimal Drift and its Surface Measure}

Since the optimal vector field $v$ is compactly supported, there is a unique solution $\left(y_{t}\right)$ of the stochastic differential equation

$$
\left\{\begin{array}{l}
d y_{t}=d b_{t}-\frac{1}{2} v\left(y_{t}\right) d t \\
y_{0}=a_{0} .
\end{array}\right.
$$

Definition 3.1. The process $\left(y_{t}\right)$ is called a Brownian motion with the optimal drift.

Let $\tau$ be the exit time of $\left(y_{t}\right)$ from $\mathbb{M}_{\varepsilon_{0}}$. Consider the stopped process $\left(y_{t \wedge \tau}\right)$ and denote by $\left(x_{t}, u_{t}, z_{t}\right)$ its Fermi decomposition. Further, consider the process

$$
\tilde{b}_{t}=\int_{0}^{t} u_{s}^{\mathrm{T}} d b_{s} .
$$

It is also an $n$-dimensional Brownian motion by the Lévy's characterization theorem since it is a continuous local martingale with $d \tilde{b}_{t}^{i} d \tilde{b}_{t}^{j}=\delta_{i j} d t$ by the orthogonality of $u_{s}$ for all $s$. Denote by $\tilde{b}_{t}^{\prime}$ (respectively, by $\tilde{b}_{t}^{\prime \prime}$ ) the first $m$ (respectively, the last $k$ ) components of $\tilde{b}_{t}$.

The following two lemmas are very similar to Lemmas 12, 13 in Ref. 6 and we skip all the computations which can be taken from their proofs.

Lemma 3.1. The Itô differential of the process $\left(x_{t}\right)$ up to time $\tau$ is given by

$$
d x_{t}=D \pi\left(y_{t}\right) u_{t} \operatorname{pr}_{1}^{-1} d \tilde{b}_{t}^{\prime}+\frac{1}{2}\left(-D \pi\left(y_{t}\right) v\left(y_{t}\right)+\Delta \pi\left(y_{t}\right)\right) d t
$$

Proof. It follows from (3.2) that

$$
d \tilde{b}_{t}^{\prime}=\operatorname{pr}_{1} u_{t}^{\mathrm{T}} d b_{t}
$$

Further, since ${ }^{6} \operatorname{Im}[D \pi(y)]=T_{\pi(y)} M$ we have

$$
D \pi\left(y_{t}\right) u_{t} \operatorname{pr}_{1}^{-1} \operatorname{pr}_{1} u_{t}^{\mathrm{T}}=D \pi\left(y_{t}\right)
$$

although $\operatorname{pr}_{1}^{-1} \mathrm{pr}_{1} \neq I$. Now by Itô's formula and using (3.1) we get up to time $\tau$

$$
\begin{aligned}
d x_{t}=d \pi\left(y_{t}\right) & =D \pi\left(y_{t}\right) d y_{t}+\frac{1}{2} D D \pi\left(y_{t}\right) d y_{t} d y_{t} \\
& =D \pi\left(y_{t}\right) u_{t} \operatorname{pr}_{1}^{-1} \operatorname{pr}_{1} u_{t}^{\mathrm{T}}\left(d b_{t}-\frac{1}{2} v\left(y_{t}\right) d t\right)+\frac{1}{2} \Delta \pi\left(y_{t}\right) d t \\
& =\pi\left(y_{t}\right) u_{t} \mathrm{pr}_{1}^{-1} d \tilde{b}_{t}^{\prime}+\frac{1}{2}\left(-D \pi\left(y_{t}\right) v\left(y_{t}\right)+\Delta \pi\left(y_{t}\right)\right) d t
\end{aligned}
$$

which completes the proof.

Lemma 3.2. $z_{t}=\tilde{b}_{t}^{\prime \prime}$ up to time $\tau$. 
Proof. Let us show that the Stratonovich differentials of the processes $\left(z_{t}\right)$ and $\left(\tilde{b}_{t}^{\prime \prime}\right)$ coincide. Denote $J_{t}=\operatorname{pr}_{2} u_{t}^{\mathrm{T}}$. It follows from Lemma 13 in Ref. 6 that

$$
\delta \tilde{b}_{t}^{\prime \prime}=J_{t} \delta b_{t}+\frac{1}{2} J_{t} d P_{x_{t}}\left(\delta x_{t}\right) \delta b_{t}
$$

Furthermore, we have

$$
d z_{t}=J_{t} \delta y_{t}=J_{t} \delta b_{t}-\frac{1}{2} J_{t} v\left(y_{t}\right) d t
$$

It remains to show that $J_{t} d P_{x_{t}}\left(\delta x_{t}\right) \delta b_{t}=-J_{t} v\left(y_{t}\right) d t$. But this is true since for each $a \in \mathbb{M}_{\varepsilon_{0}}$ the projection of $v(a)$ onto the normal space $N_{a} M$ differs from the projection of the shifting vector field ${ }^{6}$ only by the minus sign and therefore we can use the last equality from the proof of Lemma $13 .{ }^{6}$ This implies $\delta z_{t}=\delta \tilde{b}_{t}^{\prime \prime}$ and $z_{t}=\tilde{b}_{t}^{\prime \prime}$ since this stochastic differential equation is exact.

Denote by $\mathcal{L}_{y}$ the measure on $C_{a_{0}}\left([0,1], \mathbb{R}^{n}\right)$ which is the law of the process $\left(y_{t}\right)$ and define the generalized surface measure $\mathbb{S}_{y}$ corresponding to $\left(y_{t}\right)$ analogously to the definition of the generalized surface measure corresponding to a Brownian motion. Namely, we define

$$
\mathbb{W}_{P, \varepsilon}^{y}=\mathcal{L}_{y}\left(\cdot \mid \omega_{t_{i}} \in \mathbb{M}_{\varepsilon} \text { for all } t_{i} \in P\right),
$$

and

$$
\mathbb{S}^{y}=\lim _{\substack{|P| \rightarrow 0 \\ \varepsilon \rightarrow 0}} \mathbb{W}_{P, \varepsilon}^{y},
$$

where $P: 0=t_{0}<t_{1}<\cdots<t_{n-1}<t_{n}=1$ and the limit is to be understood in the weak sense.

Proposition 3.1. $\mathbb{S}^{y}=\mathbb{W}_{M}$.

Proof. It follows from Lemmas 3.1, 3.2 and (1.8) that the processes $\left(x_{t}, u_{t}, z_{t}\right)$ from the Fermi decomposition of $\left(y_{t}\right)$ satisfy (up to the exit time $\tau$ ) the following system of stochastic differential equations:

$$
\left\{\begin{array}{l}
d x_{t}=\sigma\left(x_{t}, u_{t}, z_{t}\right) d b_{t}^{\prime}+c\left(x_{t}, u_{t}, z_{t}\right) d t \\
d z_{t}=d b_{t}^{\prime \prime} \\
d u_{t}=\Gamma_{x_{t}}\left(\delta x_{t}\right),
\end{array}\right.
$$

where $\left(b_{t}^{\prime}\right)$ and $\left(b_{t}^{\prime \prime}\right)$ are two independent Brownian motions, the coefficients $\sigma$ and $c$ are given by

$$
\begin{aligned}
\sigma(x, u, z) & =D \pi\left(x+u \mathrm{pr}_{2}^{-1} z\right) u \mathrm{pr}_{1}^{-1} \\
c(x, u, z) & =-\frac{1}{2} D \pi\left(x+u \operatorname{pr}_{2}^{-1} z\right) v\left(x+u \operatorname{pr}_{2}^{-1} z\right)+\frac{1}{2} \Delta \pi\left(x+u \operatorname{pr}_{2}^{-1} z\right),
\end{aligned}
$$

and the last equation in (3.11) is just the equation of the parallel translation. 
Consider any probability space on which there is an $n$-dimensional Brownian motion $\left(b_{t}^{*}\right)$ and a family $\left(z_{t}^{P, \varepsilon}\right)$ of processes such that each $\left(z_{t}^{P, \varepsilon}\right)$ has the same law as a $k$-dimensional Brownian motion conditioned to be in the $\varepsilon$-ball at times $t_{i} \in P$, and the whole family $\left\{\left(z_{t}^{P, \varepsilon}\right)\right\}$ is independent of $\left(b_{t}^{*}\right)$. On this probability space we consider the filtration $\left(\mathcal{F}_{t}\right)$ generated by $\left\{b_{s}^{*}, s \leq t\right\}$ and all the processes $\left(z_{t}^{P, \varepsilon}\right), 0 \leq t \leq 1$. Then $\left(\operatorname{pr}_{1} b_{t}^{*}\right)$ is an $m$-dimensional $\left(\mathcal{F}_{t}\right)$-Brownian motion.

Let us show that the solution $\left(x_{t}^{P, \varepsilon}, u_{t}^{P, \varepsilon}\right)$ of the system

$$
\left\{\begin{array}{l}
d x_{t}^{P, \varepsilon}=\sigma\left(x_{t}^{P, \varepsilon}, u_{t}^{P, \varepsilon}, z_{t}^{P, \varepsilon}\right) \operatorname{pr}_{1} d b_{t}^{*}+c\left(x_{t}^{P, \varepsilon}, u_{t}^{P, \varepsilon}, z_{t}^{P, \varepsilon}\right) d t \\
d u_{t}^{P, \varepsilon}=\Gamma_{x_{t}^{P, \varepsilon}}\left(\delta x_{t}^{P, \varepsilon}\right)
\end{array}\right.
$$

converges locally uniformly in probability to the solution $\left(\bar{x}_{t}, \bar{u}_{t}\right)$ of the system

$$
\left\{\begin{array}{l}
d \bar{x}_{t}=\sigma\left(\bar{x}_{t}, \bar{u}_{t}, 0\right) \operatorname{pr}_{1} d b_{t}^{*}+c\left(\bar{x}_{t}, \bar{u}_{t}, 0\right) d t \\
d \bar{u}_{t}=\Gamma_{\bar{x}_{t}}\left(\delta \bar{x}_{t}\right)
\end{array}\right.
$$

To do this, denote the processes $\left(x_{t}^{P, \varepsilon}, u_{t}^{P, \varepsilon}\right)$ and $\left(\bar{x}_{t}, \bar{u}_{t}\right)$ by $\left(a_{t}^{P, \varepsilon}\right)$ and $\left(\bar{a}_{t}\right)$, respectively, and rewrite the systems (3.14) and (3.15) in the form

$$
d a_{t}^{P, \varepsilon}=f_{1}\left(a_{t}^{P, \varepsilon}, z_{t}^{P, \varepsilon}\right) d b_{t}^{*}+f_{2}\left(a_{t}^{P, \varepsilon}, z_{t}^{P, \varepsilon}\right) d t
$$

and

$$
d \bar{a}_{t}=f_{1}\left(\bar{a}_{t}, 0\right) d b_{t}^{*}+f_{2}\left(\bar{a}_{t}, 0\right) d t
$$

respectively, with the same initial conditions, where $f_{i}$ are the abbreviations for the coefficients in (3.14) and (3.15) and notice that

$$
f_{i}(a, z) \rightarrow f_{i}(a, 0) \text { as } z \rightarrow 0, \quad i=1,2,
$$

uniformly in $a$ and the functions $f_{i}(x, 0)$ are Lipschitz.

Now, denote

$$
\varphi_{P, \varepsilon}(t)=\mathbb{E} \sup _{s \leq t}\left\|a_{s}^{P, \varepsilon}-\bar{a}_{s}\right\|^{2}
$$

It suffices to show that $\varphi_{P, \varepsilon}(1) \rightarrow 0$ as $\varepsilon \rightarrow 0,|P| \rightarrow 0$.

Let $\delta>0$. According to the uniform convergence of $f_{i}$, choose $\varepsilon^{\prime}$ such that for all $a$ and for all $z$ with $\|z\| \leq \varepsilon^{\prime}$ holds

$$
\left\|f_{i}(a, z)-f_{i}(a, 0)\right\|<\delta, \quad i=1,2 .
$$

Further, notice that

$$
\mathbb{P}\left\{\sup _{0 \leq s \leq 1}\left\|z_{s}^{P, \varepsilon}\right\|>\varepsilon^{\prime}\right\} \rightarrow 0 \text { as } \varepsilon \rightarrow 0 \text { and }|P| \rightarrow 0
$$

since $\left(z_{t}^{P, \varepsilon}\right)$ is a conditioned Brownian motion. So there exist $\bar{\varepsilon}>0$ and $\theta>0$ such that for all $\varepsilon<\bar{\varepsilon}$ and all $P$ with $|P|<\theta$ holds

$$
\mathbb{P}\left\{\sup _{0 \leq s \leq 1}\left\|z_{s}^{P, \varepsilon}\right\|>\varepsilon^{\prime}\right\}<\delta^{2} .
$$


Using the moment estimates analogously to Lemma 16 of Ref. 6 and using additionally (3.22), the boundedness of $f_{1}, f_{2}$, and the choice of $\varepsilon^{\prime}, \bar{\varepsilon}$ and $\theta$ we get for all $\varepsilon<\bar{\varepsilon}$ and all $P$ such that $|P|<\theta$

$$
\begin{aligned}
\varphi_{P, \varepsilon}(t) \leq & c_{1} \sum_{i=1,2} \mathbb{E} \int_{0}^{t}\left\|f_{i}\left(a_{s}^{P, \varepsilon}, z_{s}^{P, \varepsilon}\right)-f_{i}\left(\bar{a}_{s}, 0\right)\right\|^{2} d s \\
\leq & c_{2} \sum_{i=1,2} \mathbb{E} \int_{0}^{t}\left\{\left\|f_{i}\left(a_{s}^{P, \varepsilon}, z_{s}^{P, \varepsilon}\right)-f_{i}\left(a_{s}^{P, \varepsilon}, 0\right)\right\|^{2}+\left\|f_{i}\left(a_{s}^{P, \varepsilon}, 0\right)-f_{i}\left(\bar{a}_{s}, 0\right)\right\|^{2}\right\} d s \\
\leq & c_{2} \sum_{i=1,2} \mathbb{E}\left(\mathbf{1}_{\left\{\sup _{0 \leq s \leq 1}\left\|z_{s}^{P, \varepsilon}\right\|>\varepsilon^{\prime}\right\}} \int_{0}^{t}\left\|f_{i}\left(a_{s}^{P, \varepsilon}, z_{s}^{P, \varepsilon}\right)-f_{i}\left(a_{s}^{P, \varepsilon}, 0\right)\right\|^{2} d s\right) \\
& +c_{2} \sum_{i=1,2} \mathbb{E}\left(\mathbf{1}_{\left\{\sup _{0 \leq s \leq 1}\left\|z_{s}^{P, \varepsilon}\right\| \leq \varepsilon^{\prime}\right\}} \int_{0}^{t}\left\|f_{i}\left(a_{s}^{P, \varepsilon}, z_{s}^{P, \varepsilon}\right)-f_{i}\left(a_{s}^{P, \varepsilon}, 0\right)\right\|^{2} d s\right) \\
& +c_{3} \mathbb{E} \int_{0}^{t}\left\|a_{u}^{P, \varepsilon}-\bar{a}_{u}\right\|^{2} d u \\
\leq & c_{4} \delta^{2}+c_{3} \int_{0}^{t} \varphi_{P, \varepsilon}(u) d u, \text { for all } t,
\end{aligned}
$$

where $c_{1}, c_{2}, c_{3}$ and $c_{4}$ are positive constants independent of $\delta$. Now by Gronwall's lemma

$$
\varphi_{P, \varepsilon}(t) \leq c_{4} \delta^{2} e^{c_{3} t}
$$

and in particular

$$
\varphi_{P, \varepsilon}(1) \leq c_{2} \delta^{2} e^{c_{3}}
$$

for all $\varepsilon<\bar{\varepsilon}$ and all $P$ such that $|P|<\theta$. Hence $\varphi_{P, \varepsilon}(1) \rightarrow 0$ and the process $\left(x_{t}^{P, \varepsilon}\right)$ converge to $\left(\bar{x}_{t}\right)$ locally uniformly in probability.

Now it remains to show that $\left(\bar{x}_{t}\right)$ is a Brownian motion on $M$. Analogously to the proof of Proposition 15 in Ref. 6 we have

$$
D \pi\left(\bar{x}_{t}\right) \bar{u}_{t} \mathrm{pr}_{1}^{-1} \operatorname{pr}_{1} d b_{t}^{*}=P_{\bar{x}_{t}} \bar{u}_{t} P_{a_{0}} d b_{t}^{*}=P_{\bar{x}_{t}}^{2} \bar{u}_{t} d b_{t}^{*}=P_{\bar{x}_{t}} d b_{t}^{* *}=D \pi\left(\bar{x}_{t}\right) d b_{t}^{* *},
$$

where $\left(b_{t}^{* *}\right)$ is another $n$-dimensional Brownian motion. Further, it follows from the proof of Lemma 2.1 that $v(x) \in N_{x} M$ for $x \in M$. This together with (3.12) and (3.13) implies

$$
\begin{aligned}
d \bar{x}_{t} & =\sigma\left(\bar{x}_{t}, \bar{u}_{t}, 0\right) \operatorname{pr}_{1} d b_{t}^{*}+c\left(\bar{x}_{t}, \bar{u}_{t}, 0\right) d t \\
& =D \pi\left(\bar{x}_{t}\right) \bar{u}_{t} \mathrm{pr}_{1}^{-1} \operatorname{pr}_{1} d b_{t}^{*}-\frac{1}{2} P_{\bar{x}_{t}} v\left(\bar{x}_{t}\right) d t+\frac{1}{2} \Delta \pi\left(\bar{x}_{t}\right) d t \\
& =D \pi\left(\bar{x}_{t}\right) d b_{t}^{* *}+\frac{1}{2} \Delta \pi\left(\bar{x}_{t}\right) d t
\end{aligned}
$$


which is equivalent to $\delta \bar{x}_{t}=P_{\bar{x}_{t}} \delta b_{t}^{* *}$ and therefore means that $\left(\bar{x}_{t}\right)$ is a Brownian motion on $M$.

\section{Surface Measure of a Standard Brownian Motion}

We are going to prove the existence of $\mathbb{S}$ and to compute its density with respect to the Wiener measure $\mathbb{W}_{M}$ using our knowledge of the measure $\mathbb{S}^{y}$. It follows from the Girsanov Theorem that the laws of $\left(b_{t}\right)$ and $\left(y_{t}\right)$ are equivalent, and the corresponding density is given by

$$
\rho=\frac{d \mathbb{W}}{d \mathcal{L}_{y}}=\exp \left\{\frac{1}{2} \int_{0}^{1}\left\langle v\left(b_{t}\right), d b_{t}\right\rangle+\frac{1}{8} \int_{0}^{1}\left\|v\left(b_{t}\right)\right\|^{2} d t\right\} .
$$

Moreover, using Itô's formula and the fact that $v$ is of the gradient type, we obtain

$$
\begin{aligned}
\rho(\omega) & =\exp \left\{\frac{1}{2} \int_{0}^{1}\left\langle\nabla \phi\left(\omega_{t}\right), d \omega_{t}\right\rangle+\frac{1}{8} \int_{0}^{1}\left\|\nabla \phi\left(\omega_{t}\right)\right\|^{2} d t\right\} \\
& =\exp \left\{\frac{\phi\left(\omega_{1}\right)-\phi\left(\omega_{0}\right)}{2}+\int_{0}^{1}\left(-\frac{\Delta \phi\left(\omega_{t}\right)}{4}+\frac{\left\|\nabla \phi\left(\omega_{t}\right)\right\|^{2}}{8}\right) d t\right\},
\end{aligned}
$$

which means that $\rho$ is continuous and bounded.

Theorem 4.1. The generalized surface measure $\mathbb{S}$ exists and is equivalent to the Wiener measure $\mathbb{W}_{M}$. The corresponding density is given by

$$
\frac{d \mathbb{S}}{d \mathbb{W}_{M}}(\omega)=C \exp \int_{0}^{1}\left(-\frac{R}{4}+\frac{m^{2}\|\kappa\|^{2}}{8}\right)\left(\omega_{t}\right) d t,
$$

where $R$ is the scalar curvature, $\kappa$ is the mean curvature vector of $M$, and $C$ is the normalizing constant.

Proof. Let $h: C_{a_{0}}\left([0,1], \mathbb{R}^{n}\right) \rightarrow \mathbb{R}$ be continuous and bounded. Denote by $\mathbb{E}, \mathbb{E}^{y}$, $\mathbb{E}_{P, \varepsilon}, \mathbb{E}_{P, \varepsilon}^{y}$, and $\mathbb{E}_{M}$ the expectations with respect to the measures $\mathbb{W}, \mathcal{L}_{y}, \mathbb{W}_{P, \varepsilon}$, $\mathbb{W}_{P, \varepsilon}^{y}$, and $\mathbb{W}_{M}$, respectively. Further, denote $\mathbf{1}_{P, \varepsilon}=\mathbf{1}_{X_{P, \varepsilon}}$, where

$$
X_{P, \varepsilon}=\left\{\omega \in C_{a_{0}}\left([0,1], \mathbb{R}^{n}\right): \omega_{t_{i}} \in \mathbb{M}_{\varepsilon} \text { for all } t_{i} \in P\right\} .
$$

By Proposition 3.1 and using the continuity and boundedness of $\rho$ we obtain

$$
\begin{aligned}
\lim _{\substack{|P| \rightarrow 0 \\
\varepsilon \rightarrow 0}} \mathbb{E}_{P, \varepsilon} h & =\lim _{\substack{|P| \rightarrow 0 \\
\varepsilon \rightarrow 0}} \frac{\mathbb{E} \mathbf{1}_{P, \varepsilon} h}{\mathbb{E} \mathbf{1}_{P, \varepsilon}}=\lim _{\substack{|P| \rightarrow 0 \\
\varepsilon \rightarrow 0}} \frac{\mathbb{E}^{y} \mathbf{1}_{P, \varepsilon} \rho h}{\mathbb{E}^{y} \mathbf{1}_{P, \varepsilon} \rho}=\lim _{\substack{|P| \rightarrow 0 \\
\varepsilon \rightarrow 0}} \frac{\mathbb{E}^{y} \mathbf{1}_{P, \varepsilon} \rho h}{\mathbb{E}^{y} \mathbf{1}_{P, \varepsilon}} \cdot \frac{\mathbb{E}^{y} \mathbf{1}_{P, \varepsilon}}{\mathbb{E}^{y} \mathbf{1}_{P, \varepsilon} \rho} \\
& =\lim _{\substack{|P| \rightarrow 0 \\
\varepsilon \rightarrow 0}} \frac{\mathbb{E}_{P, \varepsilon}^{y} \rho h}{\mathbb{E}_{P, \varepsilon}^{y} \rho}=\frac{\mathbb{E}_{M} \rho h}{\mathbb{E}_{M} \rho}=\mathbb{E}_{M}\left[h \frac{\rho}{\mathbb{E}_{M} \rho}\right] .
\end{aligned}
$$

Denote $C=\left[\mathbb{E}_{M} \rho\right]^{-1}$.

It remains to compute the restriction of $\rho$ to $M$. By Lemma 9 of Ref. 6 we have $\phi(a)=0$ for all $a \in M$ and using Lemma 2.1 we obtain 


$$
\frac{\rho}{\mathbb{E}_{M} \rho}(\omega)=C \exp \int_{0}^{1}\left(-\frac{R}{4}+\frac{m^{2}\|\kappa\|^{2}}{8}\right)\left(\omega_{t}\right) d t,
$$

for every $\omega \in C_{a_{0}}([0,1], M)$, and the theorem is proved.

\section{References}

1. O. G. Smolyanov, Smooth measures on loop groups, Dokl. Math. 52 (1995) 408-411.

2. O. G. Smolyanov, H. V. Weizsäcker and O. Wittich, Brownian motion on a manifold as limit of stepwise conditioned standard Brownian motions, Can. Math. Soc. Conf. Proc. 29 (2000) 589-602.

3. O. G. Smolyanov, H. V. Weizsäcker and O. Wittich, Diffusion on a compact Riemannian manifold and surface measures, Dokl. Math. 371 (2000) 442-447 (Russian edition).

4. N. A. Sidorova, O. G. Smolyanov, H. V. Weizsaecker and O. Wittich, Wiener surface measures on trajectories in Riemannian manifolds, Dokl. Math. 65 (2002) 234-244.

5. O. G. Smolyanov, H. V. Weizsäcker and O. Wittich, Chernoff's theorem and the construction of semigroups, Prog. Nonlinear Differential Eqs. Their Appl. 55 (2003) 349-358.

6. N. A. Sidorova, O. G. Smolyanov, H. V. Weizsaecker and O. Wittich, The surface limit of Brownian motion in tubular neighbourhoods of an embedded Riemannian manifold, J. Funct. Anal. 206 (2004) 391-413.

7. N. A. Sidorova, Brownian motion on an embedded manifold as the limit of Brownian motions with reflection in its tubular neighborhoods, Math. Notes 73 (2003) 895-899.

8. I. Davies, O. G. Smolyanov and A. Truman, Functional integral representations of solutions for stochastic Schrödinger equations on compact Riemannian manifolds, Dokl. Math. 373 (2000) 10-14 (Russian edition).

9. O. G. Smolyanov and A. Truman, Feynman-Kac formulas for solutions for Scrödinger equations on compact Riemannian manifolds, Math. Notes 68 (2000) 789-793.

10. O. G. Smolyanov and A. Truman, Feynman integrals over trajectories in Riemannian manifolds, Dokl. Math. 68 (2003) 194-198.

11. C. DeWitt-Morette, K. D. Elworthy, B. L. Nelson and G. Sammelman, A stochastic scheme for constructing solutions of the Schrödinger equations, Ann. Inst. Henri Poincaré 32 (1980) 327-341.

12. V. I. Averbukh, O. G. Smolyanov and S. V. Fomin, Generalized functions and differential equations in linear spaces I: Differentiale measures, Trans. Moscow Math. Soc. 24 (1971) 140-184.

13. H. Airault and P. Malliavin, Intégration géométrique sur l'espace de Wiener, Bull. Sci. Math. 112 (1988) 3-52.

14. R. Froese and I. Herbst, Realizing holonomic constraints in classical and quantum mechanics, Comm. Math. Phys. 220 (2001) 489-535.

15. A. V. Skorokhod, Integration in Hilbert Space (Springer, 1974).

16. A. V. Uglanov, Surface integrals in Banach spaces, Math. USSR Sbor. 38 (1981) 175-203. 